\title{
NEURITE ÓPTICA COMO MANIFESTAÇÃO DE DOENÇAS DESMIELINIZANTES E A DIFICULDADE DE IDENTIFICAÇÃO CAUSAL: RELATO DE CASO
}

\section{GUILHERME VIEIRA BORCHIO RIBEIRO ${ }^{1}$; ELIS DE OLIVEIRA CAMPOS PAIVA MOL ${ }^{2}$; VINÍCIUS PEDRO ALMEIDA VALENTIM ${ }^{3}$}

\footnotetext{
${ }^{1}$ Graduando em Medicina, Centro Universitário UniFACIG; guilhermeborchio@ yahoo.com.br.

${ }^{2}$ Mestre em Políticas Públicas e Desenvolvimento Local pela Escola Superior de Ciências da Santa Casa de Misericórdia de Vitória, Graduada em Medicina, Docente do curso de Medicina, Centro Universitário UniFACIG.

3 Especialista em Cardiologia Intervencionista e Hemodinâmica pela Fundação Santa Casa de Misericórdia de Belo Horizonte.Graduado em Medicina.
}

\section{RESUMO}

A neurite óptica é a causa mais comum de perda visual em adultos jovens, tendo em vista este fato, a realização do diagnóstico, além da determinação da causa desta afecção, é de grande importância para o manejo clínico da doença. A esclerose múltipla e a neuromielite óptica são as principais causas desmielinizantes desta doença e a distinção entre ambas se mostra muito difícil. Este artigo relata um caso, no qual há o acometimento da paciente por uma neurite óptica de causa desconhecida e mostra que, com o desenvolvimento de técnicas diagnósticas como exames laboratoriais e de imagem, juntamente com a utilização de critérios, facilitou-se a realização de tais diagnósticos.

Palavras-chave: Neurite Óptica; Esclerose Múltipla; Neuromielite Óptica; Diagnóstico.

\section{OPTIC NEURITIS AS MANIFESTATION OF DEMYELINATING DISEASES AND CAUSAL IDENTIFICATION DIFFICULTY: CASE REPORT}

\begin{abstract}
Optic neuritis is the most common cause of visual loss in young adults, in view of this fact, the accomplishment of diagnosis, besides determinig the cause of this condition, has great importance for the clinical management of the disease. Multiple sclerosis and optic neuromyelitis are the main demyelinating causes of this disease, and the distinction between both is very difficult. This article reports a case in wich the patient is affected by an optic neuritis of unknown cause, and shows that with the development of diagnostic techniques such as laboratory and imaging tests, together with the use of criteria, facilitated the accomplishment of such diagnoses.
\end{abstract}

Keywords: Optic neuritis; Multiple sclerosis; optic neuromyelitis; Diagnosis 


\section{INTRODUÇÃO}

A neurite óptica (NO) é uma inflamação de origem infecciosa, autoimune ou desmielinizante, que pode acometer um ou ambos os nervos ópticos (LOPES, 2016). Pode se manifestar isoladamente ou ser a primeira manifestação de uma doença desmielinizante como a Esclerose Multipla (EM) e a Neuromielite Óptica (NMO) (BRASIL NETO; TAKAYANAGUI, 2013).

Apesar de ser mais frequentemente associada a EM, a NO também pode estar associada a outras doenças inflamatórias e infecciosas como lúpus eritematoso sistêmico, síndrome de Sjögren, doença de Lyme, sarcoidose, sífilis e infecção pelo vírus da imunodeficiência humana (HIV) (GOLDMAN; SCHAFER, 2014).

A NMO era classificada até pouco tempo como uma variante da EM. Porém, estudos clínicos, de imagens e imuno-histoquímicos, evidenciam tratar-se de uma doença distinta (PEIXOTO et al, 2010), com fisiopatologia, evolução e tratamentos diferenciados (BRASIL NETO; TAKAYANAGUI, 2013). Isso mostra que as duas principais doenças desmielinizantes (EM e NMO) que têm a NO como manifestação clínica, são muito similares e, consequentemente, há uma dificuldade diagnóstica na diferenciação entre ambas.

Estima-se que a prevalência de NO é de 115:100.000 habitantes, com incidência de 2,6 homens e 7,5 mulheres para cada 100.000 habitantes a cada ano; sendo assim, a causa mais comum de perda visual transitória em adultos jovens (LOPES, 2016).

O presente artigo tem por objetivo relatar o caso de uma paciente com NO, cuja causa ainda não foi estabelecida, e discutir quais seriam causas e como distingui-las, com ênfase nas doenças desmielinizantes.

\section{METODOLOGIA}

O presente artigo é um estudo de relato de caso com revisão bibliográfica. Foram utilizados artigos nacionais e internacionais, encontrados em bases de dados como Scielo e Pubmed. Os critérios de inclusão foram os descritores: Neurite Óptica; Esclerose Múltipla; Neuromielite Óptica; Diagnóstico. 


\section{RELATO DE CASO}

Paciente feminina G.M.S.L., 58 anos, caucasiana, doméstica aposentada, natural e procedente de Manhuaçu-MG, casada, G2P1A1. Hipertensa controlada, em uso regular de losartan e omeprazol. Tabagista (4 anos-maço) e ex-alcoolista. Pai, também hipertenso, com história de aneurisma cerebral. Relata dor ocular e cefaleia de grande intensidade à esquerda há dois meses, uma semana após um quadro gripal. Houve uma intensificação álgica nos últimos 12 dias, seguida de piora da visão, o que a fez buscar um serviço médico. Inicialmente, foi instituído tratamento com sintomáticos e homeopatia. Sem remissão dos sintomas, passou por novas avaliações de diferentes médicos sem receber um diagnóstico preciso. Ao exame físico, foi constatado amaurose à esquerda, reflexo fotomotor direto diminuído à esquerda, e consensual diminuído à direita; hiposmia (a qual relata possuir desde o nascimento); demais pares cranianos preservados. Exames laboratoriais evidenciaram linfócitose (42,7\%); Aquaporina 4 IgG: não reagente; demais resultados dentro dos parâmetros de normalidade. Ressonância Magnética (figura 1) evidenciando assimetria da espessura dos nervos ópticos, maior à esquerda, não se observando áreas de impregnação anômalas pelo gadolínio.

Figura 1 - Ressonância magnética

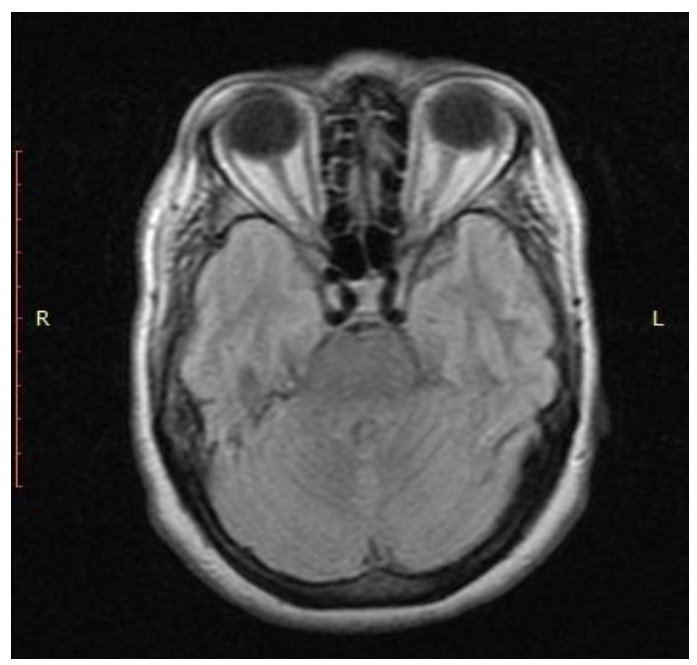

Fonte: Masterclin, 2018.

Suspeitou-se, então, de uma neurite óptica por esclerose múltipla e, por isso, foi internada na UPA de Manhuaçu para pulsoterapia com corticoides (Metilpredinisolona) 
durante quatro dias. Dez dias após a alta e término do tratamento, a paciente persiste com amaurose à esquerda e continua com o diagnóstico causal não definido, aguardando realização de ressonância magnética cervical.

\section{DISCUSSÃO}

O nervo óptico, ou II par craniano, é um nervo exclusivamente sensitivo aferente ao sistema nervoso central (SNC), que conduz os impulsos nervosos visuais oriundos da retina. Ele é constituído por um feixe espesso de fibras nervosas revestidas por mielina (MACHADO; HAERTEL, 2014).

Para que ocorra a condução do impulso nervoso de maneira adequada, é necessária a existência da bainha de mielina. Esta bainha é composta de lipídeos e é sintetizada pelos oligodendrócitos e pelas células de Schawann. Ela fornece isolamento celular e aumenta a velocidade de propagação do potencial de ação (HENRIQUES, 2014). Defeitos nesse mecanismo têm como consequência a diminuição funcional de transmissão dos impulsos, como ocorre nas doenças desmielinizantes.

A neurite óptica (NO), uma das neuropatias ópticas mais comuns em adultos, é uma manifestação comum na esclerose múltipla (EM) ou na neuromielite óptica (NMO) e pode também se apresentar isoladamente (SON et al, 2017).

Diversas causas podem ser atribuídas a NO como: doenças desmielinizantes (EM e NMO), pós-viral (mononucleose, herpes-zóster, citomegalovírus), pós-vacinal, associada a sinusite esfenoetmoidal, outras infecções (sífilis, doença de Lyme, criptococose, toxoplasmose, tuberculose), vasculites, entre outras (LOPES, 2016).

A gênese da NO se dá a partir de uma diminuição da mielina que recobre o(s) nervo(s) óptico(s) e, consequentemente, essas fibras desmielinizadas passam a conduzir os impulsos nervosos de maneira inadequada e velocidade diminuída, podendo até perder totalmente a capacidade de transmissão (LOPES, 2016). Como consequência disso, o paciente pode apresentar diminuição da acuidade visual e até amaurose.

O diagnóstico de NO desmielinizante é realizado principalmente por exclusão de outras causas de neurites e neuropatias como causas vasculares, infecciosas, por diabetes e outras doenças autoimunes (LOPES, 2016).

Existe uma correlação já estabelecida entre a EM e NO, sendo essa a alteração ocular mais importante e de maior frequência encontrada na esclerose e, muitas vezes, a 
primeira manifestação clínica da doença (SIBINELLI et al, 2000). Já a NMO, é menos comum do que a EM (menos de $1 \%$ das doenças desmielinizantes do SNC); porém, também apresenta NO como um de seus principais sintomas (PEIXOTO et al, 2010) e, por isso, suas manifestações são de difícil distinção das relativas à EM.

A EM é uma afecção de caráter autoimune e crônica, na qual se observa desmielinização, inflamação multifocal, gliose e perda de neurônios do SNC (ALVAREZ; MACÊDO; AFONSO, 2017). Do mesmo modo, a NMO, também conhecida como doença de Devic, similarmente à EM, apresenta desmielinizaçao do SNC; porém, esta afeta seletivamente os nervos ópticos e a medula espinhal (PEIXOTO et al, 2010). Este e outros critérios clínicos são usados para distinguir as duas patologias e serão mostrados adiante.

A primeira evidência a se considerar para distinguir uma possível EM ou NMO é a epidemiologia. A EM ocorre, principalmente, em adultos jovens com idade média de início entre 27 e 34 anos, raramente acometendo pessoas menores de 10 anos ou maiores de 50. Tem também uma predileção por mulheres até 2,5 maior do que homens (GOLDMAN; SCHAFER, 2014), pessoas caucasianas e habitantes de climas temperados. Já a NMO, possui um início de acometimento mais tardio (entre 35 e 40 anos), com uma maior predileção para mulheres do que a EM (8 vezes mais prevalente em mulheres); diferentemente da EM, a NMO é mais prevalente em populações asiáticas e afrodescendentes e 50\% dos casos estão associados a uma outra doença autoimune (FROTA et al, 2016). Um outro dado importante a ser discutido é que a NMO, por compreender apenas $1 \%$ de todas as doenças demielinizantes, é mais rara do que a EM (BRASIL NETO; TAKAYANAGUI, 2013)

A exposição à luz solar e elevados níveis séricos de vitamina $\mathrm{D}$ se mostraram fatores protetores para EM devido à regulação do sistema imune pelo calciferol. Já o tabagismo, foi classificado como fator de risco para a mesma doença (GOLDMAN; SCHAFER, 2014),

A EM pode ter como manifestações clínicas um único sintoma isolado ou a associação de vários deles. A paresia e a parestesia são os sintomas iniciais mais comuns da doença, evidenciados em $50 \%$ e $45 \%$ dos casos, respectivamente, seguido da NO, presente em $20 \%$ dos indivíduos, mostrando-se assim muito frequente. Manifestações cerebelares, como marcha atáxica, também podem ocorrer na EM (15\% 
dos casos), assim como outros sintomas menos comuns como: neuralgia do trigêmeo, espasmos tônicos, disfunção de esfíncteres, fadiga e distúrbios neuropsicológicos (LOPES, 2016).

A NMO pode ser definida como a associação de dois acometimentos neurológicos: a NO (já descrita anteriormente) e a Mielite transversa (MT), que podem ocorrer simultaneamente ou não (PEIXOTO et al, 2010). A MT é uma manifestação inflamatória e desmielinizante da medula espinhal de grande extensão (acomete toda a sua secção transversal ou acomete mais do que três segmentos medulares longitudinalmente) gerando sintomas de acometimento medular (GOLDMAN; SCHAFER, 2014).

Devido à falta de esclarecimento detalhado a respeito da fisiopatologia da EM, à ausência de sinais patognomônicos e à diversidade de sinais e sintomas encontrados, o diagnóstico de certeza se mostra difícil para essa doença. Todavia, a melhoria das técnicas diagnósticas atuais como a ressonância magnética e exames laboratoriais e a pesquisa de bandas oligoclonais no líquor possibilitaram a realização de diagnósticos mais assertivos na EM (LOPES, 2016).

Existem alguns critérios para diagnóstico de EM, dentre eles o critério de McDonald et al, mostrado no quadro 1, é um dos mais utilizados e mais recentes. Nele, observamos a necessidade de associar achados clínicos com achados de exames complementares para a confirmação de tal diagnóstico. Um surto é definido como um episódio de distúrbio neurológico para o qual as causas das lesões são passíveis de ser inflamatórias e desmielinizantes. Deve haver um relatório subjetivo (respaldado por achados objetivos) ou uma observação objetiva de que o evento durou pelo menos 24 horas (POLMAN et al, 2005).

Quadro 1 - Critérios diagnósticos de McDonald para Esclerose Múltipla

\begin{tabular}{|l|l|l|}
\hline Apresentação clínica & $\begin{array}{l}\text { Dados adicionais necessários ao } \\
\text { diagnóstico }\end{array}$ & \\
\hline $\begin{array}{l}\text { Dois ou mais surtos e evidências clínicas } \\
\text { de duas lesões }\end{array}$ & Nenhum & \\
\hline
\end{tabular}




\begin{tabular}{|l|l|}
\hline Dois surtos, evidência clínica de uma lesão & $\begin{array}{l}\text { Disseminação à RM* no espaço ou } \\
\text { banda oligoclonal no líquor ou novo } \\
\text { surto da doença }\end{array}$ \\
\hline $\begin{array}{l}\text { Um surto, evidência clínica de duas ou } \\
\text { mais lesões }\end{array}$ & $\begin{array}{l}\text { Disseminação à RM no tempo ou novo } \\
\text { surto da doença }\end{array}$ \\
\hline Um surto, evidência clínica de uma lesão & $\begin{array}{l}\text { Disseminação à RM no espaço ou } \\
\text { banda oligoclonal em líquor e duas ou } \\
\text { mais lesões à RM compatíveis com } \\
\text { esclerose múltipla e disseminação à } \\
\text { RNM no tempo ou um novo surto }\end{array}$ \\
\hline Progressão clínica sugestiva de EM & $\begin{array}{l}\text { Um ano de progressão da doença e dois } \\
\text { dos seguintes: nove lesões à RM, duas } \\
\text { lesões focais na medula espinhal, ou } \\
\text { banda oligoclonal em líquor. }\end{array}$ \\
\hline
\end{tabular}

* RM: Ressonância magnética

Fonte: LOPES, 2016; POLMAN et al, 2005.

É necessário entender a fisiopatologia da NMO descrita recentemente como uma canalopatia autoimune, na qual um anticorpo que se liga ao canal aquaporina-4 (AQP-4) de diversas regiões do SNC e, supostamente, causa a destruição dos astrócitos ou lesiona a barreira hematoencefálica, alterando sua permeabilidade. Como resultado desses mecanismos, ocorre um processo inflamatório desmielinizante presente na NMO. A imunoglobulina IgG encontrada no soro de pacientes com NMO que acomete esses canais foi denominada NMO-IgG e apresenta $91 \%$ de especificidade e $73 \%$ de sensibilidade para o diagnóstico dessa doença (BRASIL NETO; TAKAYANAGUI, 2013).

O exame de líquor possuía importante participação no diagnóstico de NMO; porém, com a descoberta do NMO-IgG, este não é mais um exame de tanto valor. Já a ressonância magnética, demonstra algumas diferenças entre a EM e a NMO, principalmente nas lesões medulares, as quais são extensas (maior do que três níveis vertebrais), com edema importante e evoluem com cavitação, na NMO, diferentemente da EM (BRASIL NETO; TAKAYANAGUI, 2013). 
Os critérios de Barkhoff determinam características de EM na ressonância magnética, sendo eles: 1) pelo menos uma lesão impregnada por gadolínio ou no mínimo nove lesões supratentoriais em T2; 2) no mínimo três lesões periventriculares; 3) pelo menos uma lesão justacortical; 4) uma ou mais lesões infratentoriais. A presença de, no mínimo, três das quatro características positivas sugerem EM (POLMAN et al, 2005).

Os critérios evidenciados no quadro 2, são utilizados para diagnóstico de NMO e para a confirmação são necessários a presença de todos os critérios absolutos e pelo menos dois dos critérios de suporte (BRASIL NETO; TAKAYANAGUI, 2013).

Quadro 2 - Critérios para o diagnóstico de neuromielite óptica

\begin{tabular}{|l|l|}
\hline Critérios absolutos & Critérios de suporte \\
\hline Neurite óptica & $\begin{array}{l}\text { Lesão medular maior do que três níveis } \\
\text { vertebrais }\end{array}$ \\
\hline Mielite aguda & $\begin{array}{l}\text { RM com menos de três critérios de } \\
\text { Barkhoff positivos }\end{array}$ \\
\hline $\begin{array}{l}\text { Não há limite de tempo entre a } \\
\text { ocorrência dos critérios absolutos }\end{array}$ & NMO-IgG positivo \\
\hline
\end{tabular}

RM: Ressonância magnética; NMO-IgG: anticorpo antiaquaporina 4.

Fonte: BRASIL NETO; TAKAYANAGUI, 2013.

O caso apresentado representa um quadro de NO desmielinizante sem uma causa elucidada. Epidemiologicamente, a paciente pertence a grupos de maior prevalência de EM comparados à NMO, pois é caucasiana e mora em local de clima temperado, o que fala a favor de EM e contra a NMO. Além disso, é tabagista e este é um fator de risco para EM.

A paciente do caso descrito não preenche os critérios para NMO, por não apresentar sinais de lesão medular e possível mielite; o resultado do exame para NMOIgG foi negativo, afastando assim a possibilidade de ser NMO até então. Contudo, apesar de mais provável, a paciente também não possui critérios suficientes para definir o diagnóstico de EM. Desse modo, tem-se essa NO classificada como idiopática, mas 
com risco de conversão para EM. A taxa de conversão de NO para EM para mulheres foi estimada em 13,9\% e para homens em 7,7\% (LOPES, 2016)

Por possuir uma fisiopatologia diferente da NMO, algumas imunoterapias para EM podem agravar o quadro de um paciente com NMO; portanto, o diagnóstico inicial correto é crucial para determinar o manejo da doença (CHEN et al, 2016).

\section{CONCLUSÃO}

É sabidamente difícil diagnosticar a causa de neurite óptica, especialmente diferenciar entre as duas principais afecções desmielinizantes originadoras. Porém, com o aperfeiçoamento dos exames de imagem e laboratoriais, juntamente com o uso de critérios diagnósticos, vem se tornando cada vez mais inteligível a determinação dessas doenças. Esse processo diagnóstico, se feito de maneira correta e precocemente, é de extrema importância para a evolução da doença.

\section{REFERÊNCIAS}

ALVAREZ, C.; MACÊDO, P. J. O. M.; AFONSO, C. Esclerose múltipla pseudotumoral: relato de caso e revisão de literatura. Revista Brasileira de Neurologia, v. 53, n. 1, p.38-41, 2017. Acesso em 20 de maio de 2017. Disponível em: $<$ https://revistas.ufrj.br/index.php/rbn/article/view/9543/ESCLEROSE\%20M\%C3\%9A LTIPLA\%20PSEUDOTUMORAL\%3A\%20RELATO\%20DE\%20CASO\%20E\%20RE VIS\%C3\%830\%20DE\%20LITERATURA>.

BRASIL NETO, J. P.; TAKAYANAGUI, O. M. Tratado de Neurologia da Academia Brasileira de Neurologia. 1 ed. Rio de Janeiro: Elsevier, 2013

CHEN, H. et al Clinical Features of Patients with Multiple Sclerosis and Neuromyelitis Optica Spectrum Disorders. Chinese Medical Journal, v. 129, n. 17, p. 2079-2084, 2016. Acesso em 20 de maio de 2017. Disponível em: <http://www.cmj.org/temp/ChinMedJ129172079-7356598_202605.pdf>.

FROTA, E. R. C. et al. Recomendações no tratamento da esclerose múltipla e neuromielite óptica. Academia Brasileira de Neurologia. 2 ed. São Paulo: OMNIFARMA, 2016. Acesso em 20 de maio de 2017. Disponível em: <http://formsus.datasus.gov.br/novoimgarq/28121/4880987_312361.pdf>

GOLDMAN, 1.; SCHAFER, A. I. Cecil Medicina. 24 ed. Rio de Janeiro: Elsevier, 2014. 
HENRIQUES, J. G. B. Manual de Neuroanatomia Clínica. 1 ed. Belo Horizonte: Rona, 2014

LOPES, A. C. Tratado de Clínica Médica, volume 1. 3 ed. Rio de Janeiro: Roca, 2016 MACHADO, A. B. M.; HAERTEL, L. M. Neuroanatomia Funcional. 3 ed. São Paulo: Atheneu, 2014

PEIXOTO, I. et al. Doença de Devic. Acta. Médica Portuguesa, Coimbra, v. 23, n. 2, p. 263-266, 2010. Acesso em 20 de maio de 2017. Disponível em: $<$ http://actamedicaportuguesa.com/revista/index.php/amp/article/view/607/291>.

POLMAN, C. H. et al. Diagnóstic Criteria for Multiple Sclerosis: 2005 revisions to the McDonald Criteria. Annals of Neurology, v. 58, n. 6, p. 840-846, 2005. Acesso em 20 de maio de 2017. Disponível em: <http://onlinelibrary.wiley.com/doi/10.1002/ana.20703/full\#fn2>.

SIBINELLI, M. A. M. F. et al. Manifestacões oculares em pacientes com esclerose múltipla em São Paulo. Arquivos Brasileiros de Oftalmologia, v. 63, n.4, p. 287-291, 2000. Acesso em 20 de maio de 2017. Disponível em: <http://www.scielo.br/pdf/abo/v63n4/12333>.

SON, D. Y. et al. Initial Pattern of Optic Nerve Enhancement in Korean Patients with Unilateral Optic Neuritis. Korean J Ophthalmological, v. 31, n. 1, p. 71-79, 2017. Acesso em 20 de maio de 2017. Disponível em: <https://synapse.koreamed.org/DOIx.php?id=10.3341/kjo.2017.31.1.71>. 